\title{
On Thabit ibn Kurrah's Formula for Amicable Numbers
}

\author{
By Walter Borho
}

\begin{abstract}
This note describes some methods for deriving explicit formulas for amicable numbers which are similar to Thabit's famous rule. The search for new amicable pairs by such" rules consists merely in primality tests for certain big numbers. Only two new pairs are actually given, demonstrating the usefulness of the results and simultaneously illustrating the quite strange form of pairs obtained by this new method. It is proposed to use a computer in order to get more solutions.
\end{abstract}

1. The numbers 220,284 seem to have been known already to Pythagoras as the smallest pair of amicable numbers. They represent the case $n=2$ of the following formula due to Thabit ibn Kurrah, an Arabian mathematician of the 9th century: If $p=3 \cdot 2^{n-1}-1, q=3 \cdot 2^{n}-1$, and $r=9 \cdot 2^{2 n-1}-1$ are primes and $n \geqq 2$, then $2^{n} p q$ and $2^{n} r$ are amicable numbers [4].

This theorem was rediscovered by Fermat (1636) and Descartes (1638) and generalized by Euler: $2^{n} p q$ and $2^{n} r$ are amicable numbers, if the three integers $p=2^{n-m} f-1$ $q=2^{n} f-1$, and $r=2^{2 n-m} f^{2}-1$ are primes, where $n>m \geqq 1$ and $f=2^{m}+1$.

Thabit's rule yields amicable numbers for $n=2,4,7$, but for no other value $n<$ 200. With Euler's generalization, there is still one more solution: $m=7, n=8$ (Legendre, Tchebychev). Whether there are any further solutions is not known.* In particular, it is an open question, whether there is an infinity of amicable pairs of numbers, each having at most three distinct prime divisors (but cf. [6]).

Apparently it has not been noticed up to now, that various rules can be found which are quite analogous to Thabit's. They arise naturally on considering the question whether there exists an infinity of amicable pairs with a given number of distinct prime divisors (cf. also [2]).

Notation. $\sigma(n)$ denotes the sum of all, $\tau(n)$ the sum of all proper divisors of $n$, i.e., $\sigma(n)=n+\tau(n)$.

2. By a "Thabit-ibn-Kurrah-rule" or "Thabit-rule" $T\left(b_{1}, b_{2}, p ; F_{1}, F_{2}\right)$, with given natural numbers $b_{1}, b_{2}$, a prime $p$, and polynomials $F_{1}(X), F_{2}(X) \in \mathrm{Z}[X]$, we mean a statement of the form:

$$
\begin{aligned}
& p^{n} b_{1} F_{1}\left(p^{n}\right) \text { and } p^{n} b_{2} F_{2}\left(p^{n}\right) \text { are amicable numbers, if } \\
& F_{i}\left(p^{n}\right) \text { is prime and prime to } b_{i} p \text { for } i=1,2 .
\end{aligned}
$$

The following Lemma yields the key for the consideration of such "rules":

Received September 7, 1971, revised November 4, 1971.

AMS 1970 subject classifications. Primary 10A40; Secondary 10A25.

$K e y$ words and phrases. Amicable numbers, amicable $k$-cycles, primality tests.

* In a letter, E. J. Lee pointed out that it can be seen from H. Riesel's tables in Math. Comp., v. 23,1969 , pp. $869-875$, that there is no further solution for $200 \leqq n \leqq 1000$. 
LemMA. Let $b_{1}, b_{2}$ be positive integers, $p$ a prime not dividing $b_{1}, b_{2}$. Let $\mathfrak{M}$ denote the set of amicable pairs $\left(m_{1}, m_{2}\right)$ of the form $m_{i}=p^{n} b_{i} q_{i}(i=1,2)$, with $q_{1}, q_{2}$ prime and $n$ natural. Then, a necessary condition for $\mathfrak{M}$ to be infinite is

$$
\frac{p}{p-1}=\frac{b_{1}}{\sigma\left(b_{1}\right)}+\frac{b_{2}}{\sigma\left(b_{2}\right)}
$$

Proof. First, we note that, for $\left(m_{1}, m_{2}\right) \in \mathfrak{M}$, from

$$
\begin{aligned}
\sigma\left(m_{i}\right) & =m_{1}+m_{2}=p^{n}\left(b_{1} q_{1}+b_{2} q_{2}\right), \\
\sigma\left(m_{i}\right) & =\sigma\left(p^{n}\right) \sigma\left(b_{i} q_{i}\right) \quad \text { if } q_{i} \neq p \\
& =\sigma\left(p^{n+1}\right) \sigma\left(b_{i}\right) \quad \text { if } q_{i}=p,
\end{aligned}
$$

it follows that always

$$
\sigma\left(b_{i} q_{i}\right) \equiv 0\left(\bmod p^{n}\right)
$$

for $i=1,2$.

We claim that for all but at most finitely many pairs $\left(m_{1}, m_{2}\right) \in \mathfrak{M}, q_{i} \nmid b_{i} p$ for $i=1,2$. Indeed, if $q_{1}$ or $q_{2}$ is one of the (finitely many) prime divisors of $b_{1} b_{2} p$, then the possible values for $n$ are bounded by (2), and one of the $q_{i}$, together with $n$, determines the pair $\left(m_{1}, m_{2}\right)$.

Now, we have, for almost all $\left(m_{1}, m_{2}\right) \in \mathfrak{M}$,

$$
\sigma\left(m_{i}\right)=\sigma\left(p^{n} b_{i}\right)\left(q_{i}+1\right)=p^{n} b_{1} q_{1}+p^{n} b_{2} q_{2}=m_{1}+m_{2} \quad(i=1,2),
$$

which, for given $n$, is a nonsingular linear equation system determining $q_{1}, q_{2}$ uniquely. From (2), it follows that $q_{i}(n) \rightarrow \infty$ if $n \rightarrow \infty$. Now, the equation

$$
1=\frac{m_{1}}{\sigma\left(m_{1}\right)}+\frac{m_{2}}{\sigma\left(m_{2}\right)}=\sum_{i=1,2} \frac{b_{i}}{\sigma\left(b_{i}\right)} \frac{p^{n}}{\sigma\left(p^{n}\right)} \frac{q_{i}}{q_{i}+1}
$$

yields (1), if we take the limits $n \rightarrow \infty$ on the right side. Q.E.D.

Conversely, we now suppose that $b_{1}, b_{2}$ and $p$ are any numbers given as in the Lemma and satisfying condition (1). We ask for the amicable pairs in $\mathfrak{M}$ with $q_{i} \nmid b_{1} p$ $(i=1,2)$. They can be found by merely solving the system (3) for $q_{1}, q_{2}$. This yields

THEOREM 1. Let $b_{1}, b_{2}$ be natural numbers and $p \nmid b_{1} b_{2} a$ prime with property (1). If for some $n \geqq 1$, both numbers

$$
q_{i}=p^{n}(p-1)\left(b_{1}+b_{2}\right) / \sigma\left(b_{i}\right)-1, \quad i=1,2,
$$

are prime integers not dividing $b_{i} p$, then $m_{i}=p^{n} b_{i} q_{i}(i=1,2)$ are amicables.

Remark. Let $b_{1}, b_{2}$ be given as in Theorem 1 , and suppose that the theorem yields any amicable pair with these given values of $b_{1}, b_{2}$. Then, $b_{1}$ and $b_{2}$ must contain the prime 2 in the same power. This is easy to prove (cf. [2] for a more general statement).

3. The following examples will demonstrate how we can deduce Thabit-rules from this theorem.

In this section, we start from a pair $b_{1}, b_{2}$ of the special form $b_{1}=a u, b_{2}=a$ with $(a, u)=1$. We have to choose $a$ and $u$ such that (1) determines a prime, $p$. Eqs. (1) and (4) now read: 


$$
\begin{aligned}
\frac{p}{p-1} & =\frac{a}{\sigma(a)} \frac{u+\sigma(u)}{\sigma(u)}, \\
q_{1} & =p^{n}(p-1) \frac{a}{\sigma(a)} \frac{u+1}{\sigma(u)}-1=p^{n+1} \frac{u+1}{u+\sigma(u)}-1 .
\end{aligned}
$$

If, for instance, $(u+1, u+\sigma(u))=1$, then, $q_{1}$ is an integer if and only if $p=$ $u+\sigma(u)$. Hence, by (5), $\sigma(a) / a=(p-1) / \sigma(u)$, and

$$
q_{1}=p^{n}(u+1)-1, \quad q_{2}=p^{n} \sigma(u)(u+1)-1 .
$$

So we arrive at

THEOREM 2. Choose a positive number $u$ such that

$$
p=u+\sigma(u)
$$

becomes a prime. Determine a positive number a prime to up as a solution of

$$
\sigma(a) / a=(p-1) / \sigma(u) .
$$

Then, $T(a u, a, p,(u+1) X-1,(u+1) \sigma(u) X-1)$ is a Thabit-rule.

Now, we must find solutions of the system (6), (7). Of course, we can do this by testing small values of $u$. With $u=1$, we have $p=2, a=1$, and the theorem becomes the well-known rule of Euclid for even perfect numbers.

Observe that Theorem 2 cannot yield any amicable pair, if $u$ is even, or a square $>1$, or prime, or three times a prime, or if $(u, \sigma(u)) \neq 1$. That $u$ cannot be even follows from the remark in Section 2. If $u$ is a square, then both $u$ and $\sigma(u)$ are odd. Then, $p$ must be even by (6). Hence, $p=2$ and $u=1$. If $u$ is a prime, then it follows from (6) and (7) that $\sigma(a u)=\sigma(a)(u+1)=2 a u$. This means that $a u$ is a perfect number. If now $m_{1}=a u p^{n} q_{1}$ and $m_{2}=a p^{n} q_{2}$ are amicable and $\left(p q_{1}, a u\right)=1$, then $a u$ evidently divides $m_{2}$. Hence, both $m_{1}$ and $m_{2}$ are abundant numbers, which is a contradiction, since they are amicable.-If $3 \mid u$, then necessarily $\sigma(u) \equiv 1(3)$, since otherwise one of $p, q_{1}, q_{2}$ becomes divisible by 3 and hence is not prime. If, furthermore, $u=3 r$, $r$ a prime $>3$, we deduce from $\sigma(u) \equiv 1(3)$ the contradiction $3 \mid r$. - Finally, $(u, \sigma(u))$ $=1$ holds, since $p=u+\sigma(u)$ is prime.-According to these observations, the least values of $u$ which we have to test are $u=5 \cdot 7=35$ and $u=5 \cdot 11=55$.

Example. Putting $u=5 \cdot 11$, we have $p=5 \cdot 11+6 \cdot 12=127$ and $\sigma(a) / a=7 / 4$ by (6) and (7). The equation for $a$ has the (unique) solution $a=2^{2}$. Hence, the rule $T\left(2^{2} \cdot 5 \cdot 11,2^{2}, 127,56 X-1,56 \cdot 72 X-1\right)$ holds. In other words, $2^{2} \cdot 127^{n} \cdot 5 \cdot 11 \cdot q_{1}$ and $2^{2} \cdot 127^{n} \cdot q_{2}$ are amicable, if $q_{1}=56 \cdot 127^{n}-1$ and $q_{2}=56 \cdot 72 \cdot 127^{n}-1$ are prime. -With $n=2$, we obtain the new amicable pair

$$
2^{2} \cdot 127^{2} \cdot 5 \cdot 11 \cdot 903223 \text { and } 2^{2} \cdot 127^{2} \cdot 65032127 \text {. }
$$

Note that one of these numbers is divisible by 220, the smaller member of Pythagoras' pair mentioned in the introduction. As we shall see soon, this is by no means an accident.

4. Now, we shall show how to solve Eqs. (6) and (7) of Theorem 2 in a more elegant way than by trial and error. Eliminating $p$ and putting $s=\sigma(u)-1$, we obtain

$$
\sigma(a u)=\sigma(a) \sigma(u)=\sigma(a)(s+1)=a u+a s .
$$


If we suppose that $s$ is a prime not dividing $a$, then (9) is just the statement that $a u$ and as are amicable numbers. So we make the striking observation that we can gain Thabit-rules starting from certain already known amicable numbers.

THEOREM 3. Let au, as be an amicable pair with $(u s, a)=1$ and s a prime. If $p=$ $u+\sigma(u)$ is a prime not in $a$, then the following Thabit-rule holds:

$$
\begin{aligned}
& m_{1}=a u p^{n} q_{1} \text { and } m_{2}=a p^{n} q_{2} \text { are amicable if } q_{1}=p^{n}(u+1)-1 \\
& \text { and } q_{2}=p^{n}(u+1) \sigma(u)-1 \text { are primes not dividing } a \text {. }
\end{aligned}
$$

If we take, for $a u, a s$, Pythagoras' pair 220,284 , the theorem is applicable and we reobtain the example of Section 3.

There are at least 64 known amicable pairs au, as of the form needed in Theorem 3. An inspection of the smaller of these pairs produced primes $p=u+\sigma(u)=$ $u+s+1$ in 15 cases and hence 15 Thabit-rules. They are listed in Table 1 . The last

\section{TABLE 1}

Thabit-ibn-Kurrah-rules $T(a u, a, p,(u+1) X-1,(u+1) \sigma(u) X-1)$

\begin{tabular}{|c|c|c|c|c|c|}
\hline No. & $a$ & $u$ & $p$ & $\sigma(u)$ & $\begin{array}{l}\text { rule is obtained } \\
\text { from pair No. }\end{array}$ \\
\hline 1 & $2^{2}$ & $5 \cdot 11$ & 127 & 72 & (1) of [4] \\
\hline 2 & $3^{2} \cdot 7 \cdot 13$ & $5 \cdot 17$ & 193 & 108 & (10) of [4] \\
\hline 3 & $3^{2} \cdot 5 \cdot 13$ & $11 \cdot 19$ & 449 & 240 & (7) of [4] \\
\hline 4 & $3^{2} \cdot 7^{2} \cdot 13$ & $5 \cdot 41$ & 457 & 252 & (13) of [4] \\
\hline 5 & $3^{2} \cdot 7^{2} \cdot 13 \cdot 97$ & $5 \cdot 193$ & 2129 & 1164 & (14) of [4] \\
\hline 6 & $3^{4} \cdot 5 \cdot 11$ & $29 \cdot 89$ & 5281 & 2700 & (15) of [4] \\
\hline 7 & $3^{2} \cdot 7 \cdot 13 \cdot 41 \cdot 163$ & $5 \cdot 977$ & 10753 & 5868 & (11) of [4] \\
\hline 8 & $3^{2} \cdot 5 \cdot 19 \cdot 37$ & $7 \cdot 887$ & 13313 & 7104 & (9) of [4] \\
\hline 9 & $3^{4} \cdot 7 \cdot 11 \cdot 29$ & $13 \cdot 521$ & 14081 & 7308 & (3) of [1], Wulf \\
\hline 10 & $3^{2} \cdot 5 \cdot 13 \cdot 19$ & $29 \cdot 569$ & 33601 & 17100 & (8) of [4] \\
\hline 11 & $3^{2} \cdot 7^{2} \cdot 13$ & $5 \cdot 53 \cdot 97$ & 57457 & 31752 & (37) of [4] \\
\hline 12 & $3^{2} \cdot 5^{2} \cdot 13.31$ & $149 \cdot 449$ & 134401 & 67500 & (21) of [4] \\
\hline 13 & $3^{3} \cdot 5^{3} \cdot 13$ & $149 \cdot 449$ & 134401 & 67500 & (22) of [4] \\
\hline 14 & $2 \cdot 7^{2} \cdot 19 \cdot 23$ & $11 \cdot 13523$ & 311041 & 162288 & (3) of [5] \\
\hline 15 & $3^{4} \cdot 5 \cdot 11 \cdot 59$ & $89 \cdot 5309$ & 950401 & 477900 & (23) of [4] \\
\hline
\end{tabular}
obtained by applying Theorem 3

column of the table gives the number of the pair au, as in the numeration of Escott [4] or others. - Theorem 3 is applicable to any amicable pair resulting from its application. But this is of purely theoretical interest, since the arising values of $q_{1}, q_{2}$ become too large for the available primality tests.

Now we return to Eq. (9) and note that it is actually irrelevant for our purposes whether $s$ is prime (and hence $a u$ and $a s$ amicable) or not. Should we restrict ourselves to Theorem 3, then we should not get those Thabit-rules of Theorem 2 for which only $p=u+\sigma(u)$ and not $s=\sigma(u)-1$ is a prime.

But these cases may yield to some of the known methods for constructing amicable 
pairs. For instance, put $u=\delta r_{1} r_{2}$, assume $a$ and $v$ are given, and ask for suitable values for the primes $r_{1}, r_{2}$. Then, apply E. J. Lee's bilinear diophantine equation method (BDE method for short) [7], which may be stated as follows: With given naturals $a_{1}, a_{2}$, form

$$
\begin{aligned}
& F=\tau\left(a_{2}\right) \sigma\left(a_{1}\right), \quad G .=a_{2} \sigma\left(a_{2}\right), \\
& D .=a_{1} a_{2}-\tau\left(a_{1}\right) \tau\left(a_{2}\right),
\end{aligned}
$$

and take any decomposition of $(F+D) F+D G=D_{1} D_{2}$ into two natural factors $D_{1}, D_{2}$. If then

$$
r_{i}=\left(D_{i}+F\right) / D \quad(i=1,2)
$$

and

$$
s=\frac{\sigma\left(a_{1}\right)}{\sigma\left(a_{2}\right)}\left(r_{1}+1\right)\left(r_{2}+1\right)-1
$$

are primes and $\left(r_{1}, r_{2}\right)=\left(r_{1} r_{2}, a_{1}\right)=\left(s, a_{2}\right)=1$, then the numbers $a_{1} r_{1} r_{2}$ and $a_{2} s$ are amicable.

Now, for our purposes, we put $a_{1}=a v$ and $a_{2}=a$, and check only whether two numbers $r_{1}, r_{2}$ formed as in the above procedure are prime, distinct, and prime to $a_{1}$. Regardless of $s$ being prime or not, we test whether

$$
p=u+\sigma(u)=v r_{1} r_{2}+\sigma(v)\left(r_{1}+1\right)\left(r_{2}+1\right)
$$

is a prime and $p \nmid a$. If such is the case, then we have found a Thabit-rule of the form treated in Theorem 2.

As an application of the method just described, we put $v=1$, that is $u=r_{1} r_{2}$, $r_{1} \neq r_{2}$. (We have seen in Section 3, that this is the simplest possibility for the prime decomposition of $u$.) It then follows that Eq. (7) of Theorem 2 is satisfied if and only if

$$
r_{i}=\left(d_{i}+\tau(a)\right) /(a-\tau(a)) \quad(i=1,2),
$$

where $d_{1} d_{2}=a^{2}$. So we get the following:

THEOREM 4. Choose any natural $a$ and a factorization $d_{1} d_{2}=a^{2}$ of $a^{2}$, such that $r_{1}, r_{2}$, determined by (10), are distinct primes not dividing $a$. If $p=2 r_{1} r_{2}+r_{1}+r_{2}+1$ is a prime, $p \nmid a$, then the following rule holds:

$$
m_{1}=a p^{n} r_{1} r_{2} q_{1} \text { and } m_{2}=a p^{n} q_{2}
$$

are amicable if

$$
q_{1}=\left(r_{1} r_{2}+1\right) p^{n}-1 \text { and } q_{2}=\left(r_{1} r_{2}+1\right)\left(r_{1}+1\right)\left(r_{2}+1\right) p^{n}-1
$$

are primes not dividing $a$.

Putting $a=2^{m}$, we obtain the special case:

THEOREM 5. Let $k, m, n$ be naturals, $k<m$, and put $f=2^{k}+1, g=2^{m-k} f^{2}$. If all the following five numbers

$$
\begin{gathered}
r_{1}=f \cdot 2^{m-k}-1, \quad r_{2}=f \cdot 2^{m}-1, \\
p=g\left(2^{m+1}-1\right)+1, \quad q_{1}=p^{n}\left[\left(2^{m}-1\right) g+2\right]-1, \\
q_{2}=2^{m} p^{n} g\left[\left(2^{m}-1\right) g+2\right]-1
\end{gathered}
$$


are primes, then

$$
m_{1}=2^{m} p^{n} r_{1} r_{2} q_{1} \text { and } m_{2}=2^{m} p^{n} q_{2}
$$

are amicable numbers.

With $k=1, m=2, n=2$, the above five numbers are indeed primes. The resulting amicable numbers are pair (8) again. With $k=1, m=2$ and with $k=1, m=3$, Theorem 5 yields Thabit-rules. The first is of the type considered in Theorem 3 , the second is not (since $s=287$ is not a prime).

5. Returning to Theorem 1 , we now leave the restrictions made at the beginning of Section 3, and put, more generally, $b_{1}=a u, b_{2}=a v$ with $(a, u v)=1$. Then condition (1) can be given the form

$$
\frac{\sigma(a)}{a} \cdot \frac{p}{p-1}=\frac{u}{\sigma(u)}+\frac{v}{\sigma(v)} .
$$

To solve this equation by trial and error, it is convenient to proceed as follows: Choose special small values for $u$ and $v$. Then, since $p \nmid a, p$ must be one of the prime divisors of the numerator on the right side of (11). Take one of these values for $p$, and, if possible, solve the remaining equation for $a$. Finally, look whether the solution of (1) thus found yields a Thabit-rule with Theorem 1.

Three of the rules in Table 2 have been found in this way.

\section{TABLB 2}

Thabit-ibn-Kurrah-rules $T\left(b_{1}, b_{2}, p, F_{1}, F_{2}\right)$ as defined in Section 2, which are not obtained by Theorem 3

\begin{tabular}{llllrrrl}
\hline No. & \multicolumn{1}{c}{$a$} & \multicolumn{1}{c}{$b_{1}$} & $b_{2}$ & $p$ & \multicolumn{1}{c}{$F_{1}$} & \multicolumn{1}{c}{$F_{2}$} & obtained by \\
\hline 16 & $3^{2} \cdot 5 \cdot 13$ & $a \cdot 29$ & $a \cdot 11$ & 113 & $80 X-1$ & $200 X-1$ & Section 5 \\
17 & $2 \cdot 7$ & $a \cdot 5 \cdot 13$ & $a \cdot 17$ & 433 & $246 X-1$ & $1148 X-1$ & Section 5 \\
18 & $2 \cdot 5$ & $a \cdot 23 \cdot 29$ & $a \cdot 7$ & 1297 & $674 X-1$ & $60660 X-1$ & Section 5 \\
19 & $2^{3} !$ & $a \cdot 11 \cdot 23$ & $a$ & 541 & $254 X-1$ & $73152 X-1$ & Theorem 5 \\
20 & $3^{2} \cdot 5 \cdot 13 \cdot 19$ & $a \cdot 37 \cdot 113$ & $a$ & 8513 & $4182 X-1$ & $18116424 X-1$ & Theorem 4 \\
\hline
\end{tabular}

One remark is in order: If one has found any Thabit-rule $T\left(b_{1}, b_{2}, p, F_{1}, F_{2}\right)$, then it may happen that one of the congruences

$$
F_{i}\left(p^{n}\right) \equiv 0(\bmod r)
$$

for $i=1$ or 2 , is satisfied identically for a prime, $r$. Such rules have, of course, been deleted from our list, since it is evident that they cannot produce more than (at most) one amicable pair.

6. In the last two sections, we leave the Thabit-rules in the narrow sense of Section 2 and demonstrate how to use the above ideas for two other purposes. In Section 6, we shall combine the Thabit-rule method with Lee's BDE method to get a new method for the constructive search for amicable pairs, while, in Section 7, we 
shall deal with analogues of Thabit-rules in the case of amicable 4-cycles. For shortness, we only calculate representative examples and omit the details.

We ask for amicable pairs of the form $m_{1}=p^{n} b_{1} q, m_{2}=p^{n} b_{2} r_{1} r_{2}$ with $b_{1}, b_{2}$ given, $n$ variable, and $p, q, r_{1}, r_{2}$ unknown primes. This means that we replace the prime $q_{2}$ of our former considerations by the product of two primes $r_{1}, r_{2}$. We demand that $b_{1}, b_{2}$ and $p$ satisfy condition (1). If we put $b_{1}=a u, b_{2}=a v$ with $(a, u v)=1$, then (1) becomes (11).

Example 1. Take $u=53, v=5$. Then,

$$
\frac{\sigma(a)}{a}=\frac{p-1}{p} \cdot \frac{7^{2}}{3^{3}}
$$

It follows that $p=7$, and then that $a=3^{2} \cdot 13$. So we look for amicables

$$
m_{1}=3^{2} \cdot 7^{n} \cdot 13 \cdot 53 \cdot q, \quad m_{2}=3^{2} \cdot 7^{n} \cdot 13 \cdot 5 \cdot r_{1} r_{2} \quad(n=1,2,3, \cdots) .
$$

Applying the BDE method as stated in Section 4 , we find that $m_{1}, m_{2}$ are amicable if (and only if)

$$
r_{i}=\frac{1}{2}\left(d_{i}+45 \cdot 7^{n-1}\right)-1 \quad(i=1,2)
$$

and

$$
q=\frac{1}{8}\left(r_{1}+1\right)\left(r_{2}+1\right)-1
$$

are prime integers, where

$$
d_{1} d_{2}=3^{3} \cdot 7^{n-1}\left(75 \cdot 7^{n-1}+32\right) .
$$

With $n=2$, this yields the new amicable pair

$$
m_{1}=3^{2} \cdot 7^{2} \cdot 13 \cdot 53 \cdot 49727, \quad m_{2}=3^{2} \cdot 7^{2} \cdot 13 \cdot 5 \cdot 167 \cdot 2663 \text {. }
$$

Example 2. $m_{1}=2 \cdot 5^{n} \cdot 7 \cdot q$ and $m_{2}=2 \cdot 5^{n} \cdot r_{1} r_{2}$ are amicable, if $n$ is natural, the three numbers

$$
\begin{aligned}
& r_{i}=\frac{1}{3}\left(8 \cdot 5^{n}+d_{i}\right)-1 \quad(i=1,2) \\
& q=\frac{1}{8}\left(r_{1}+1\right)\left(r_{2}+1\right)-1
\end{aligned}
$$

are integer and prime, and

$$
d_{1} d_{2}=2^{4} \cdot 5^{n}\left(4 \cdot 5^{n}+9\right) .
$$

7. Let $\tau^{(k)}$ denote the $k$-fold iteration of $\tau$. If $\tau^{(k)}(n)=n$ and $\tau^{(i)}(n) \neq n$ for $1 \leqq i<k$, then $\tau(n), \cdots, \tau^{(k)}(n)$ is called an amicable (or "sociable") $k$-cycle. This is an old generalization of amicable numbers $(k=2)$ recently treated again in [1] and [3]. In [1], the following method for constructing amicable 4-cycles is given:

THEOREM. Let $a_{i}, d_{i}(i=1,2)$ be given naturals with $a_{1} \neq a_{2}, a_{1} a_{2}=d_{1} d_{2}$. Let $D=a_{1} a_{2}-\tau\left(a_{1}\right) \tau\left(a_{2}\right)$,

$$
\begin{aligned}
p_{i i} & =D^{-1}\left[\tau\left(a_{i+1}\right) \sigma\left(a_{i}\right)+d_{i} \sigma\left(a_{i+1}\right)\right], \\
r_{i} & =a_{i}^{-1} \tau\left(a_{i} p_{1 i} p_{2 i}\right),
\end{aligned}
$$

where $i, j=1,2$ and $a_{3}:=a_{1}$. If then the six numbers $p_{i i}, r_{i}$ are primes and $p_{i i} \nmid a_{i}$, $r_{i} \nmid a_{i}, p_{1 i} \neq p_{2 i}$ for $i, j=1,2$, then the four numbers

$$
n_{2 i-1}=a_{1} p_{1 i} p_{2 i}, \quad n_{2 i}=a_{i} r_{i} \quad(i=1,2)
$$


form an amicable 4-cycle. For the $p_{i i}$ to be integer, a necessary condition is

$$
\left(a_{1}-a_{2}\right)\left(\sigma\left(a_{1}\right), \sigma\left(a_{2}\right)\right) \equiv 0(\bmod D) .
$$

For our present intention, we take $a_{i}$ in the form $a_{i}=p^{n} b_{i}(i=1,2)$ with a triple $b_{1}, b_{2}, p$ ( $p$ prime and prime to $b_{1} b_{2}$ ), solving Eq. (1) with $n$ variable. We check condition (12) and then try to apply the theorem. ((12) is automatically satisfied if, for instance, $b_{1}=a u, b_{2}=a$, with $a, u$ as in Theorem 2.)

Example. $a_{1}=2 \cdot 5 \cdot 7^{n}, a_{2}=2 \cdot 11 \cdot 7^{n}$ satisfy all of our conditions. The theorem yields: The four numbers

$$
\begin{array}{ll}
n_{1}=2 \cdot 5 \cdot 7^{n} \cdot p_{11} p_{21}, & n_{2}=2 \cdot 5 \cdot 7^{n} \cdot r_{1}, \\
n_{3}=2 \cdot 11 \cdot 7^{n} \cdot p_{21} p_{22}, & n_{4}=2 \cdot 11 \cdot 7^{n} \cdot r_{2}
\end{array}
$$

form an amicable 4-cycle, if the six numbers

$$
\begin{gathered}
p_{11}=\frac{2}{3}\left(7^{\lambda}+5 \cdot 7^{n}\right)-1, \quad p_{21}=\frac{10}{3}\left(11 \cdot 7^{2 n-\lambda}+7^{n}\right)-1, \\
p_{12}=\frac{1}{3}\left(7^{\lambda}+11 \cdot 7^{n}\right)-1, \quad p_{22}=\frac{11}{3}\left(5 \cdot 7^{2 n-\lambda}+7^{n}\right)-1, \\
r_{i}=\frac{1}{a_{i}} \tau\left(n_{2 i-1}\right) \quad(i=1,2)
\end{gathered}
$$

are primes. Here, $\lambda$ is an integer with $0 \leqq \lambda \leqq 2 n$. The author does not know, whether this example actually yields a new amicable 4-cycle.

Note that the point of our method is that the denominators $D$ of Sections 6-7 can be made independent of $p^{n}$ by use of condition (1). Many examples similar to those given in Sections 6 and 7 can be found easily.

For a Thabit-rule like formula for amicable 3-cycles, the interested reader is referred to [1].

Added in proof. E. J. Lee tested by computer several of the rules given in Tables 1 and 2 for some small values of the exponent $n$. In a letter dated August 20, 1971, he announced primality of $q_{1}, q_{2}$ in the case $n=1$ of rule no. 6 . This means discovery of the new amicable pair

$$
3^{4} \cdot 5 \cdot 11 \cdot 5281 \cdot 29 \cdot 89 \cdot 13635541 \text { and } 3^{4} \cdot 5 \cdot 11 \cdot 5281 \cdot 36815963399 \text {. }
$$

Essener Str. 13f

2 Hamburg 62

West Germany

1. W. BoRHO, Uber die Fixpunkte der $k$-fach iterierten Teilersummenfunktion," Mitt. Math. Gesellsch. Hamburg, v. 9, 1969, no. 5, pp. 34-48. MR 40 \#189.;

2. W. BoRHO, "Befreundete Zahlen mit gegebener Primteileranzahl." (To appear.)

3. H. COHEN, "On amicable and sociable numbers," Math. Comp., v. 24, 1970, pp. $423-$ 429. MR 42 \#587.

4. E. B. EscotT, "Amicable numbers," Scripta Math., v. 12, 1946, pp. 61-72. MR 8, 135. \#5158.

5. M. GARCIA, "New amicable pairs," Scripta Math., v. 23, 1957, pp. 167-171. MR 20

6. H.J. Kanold, "Über befreundete Zahlen. II," "Math. Nachr., v. 10, 1953, pp. 99-111. MR 15, 506 .

7. E. J. LEE, "Amicable numbers and the bilinear diophantine equation," Math. Comp., v. 22, 1968, pp. 181-187. MR 37 \#142. 